\title{
Type IV collagen and laminin staining patterns in benign and malignant cutaneous lesions
}

\author{
RONA M MacKIE, D B CLELLAND, CHRISTINE J SKERROW \\ From the Department of Dermatology, University of Glasgow, Scotland
}

SUMMARY The presence of both laminin and type IV collagen was sought at the dermo-epidermal junction and in the dermis adjacent to benign melanocytic naevi of the junctional, compound, and intradermal types; dysplastic naevi; and both primary and secondary melanoma. In all, 154 lesions were studied, using antibodies to laminin and type IV collagen and an indirect immunoperoxidase technique. The staining patterns seen with the two antibodies were virtually identical, although that of laminin was generally fainter. Breaks in and thinning of the normally continuous line of type IV collagen and laminin at the dermo-epidermal junction were seen in association with the junctional activity of benign naevi, and in malignant melanomas in association with invasive tumour cells. Both benign and malignant cells of the melanocyte series showed relatively light pericellular staining around individual cells and clusters of cells in the papillary dermis. This staining pattern was much stronger in the deeper reticular dermis.

It is concluded that the pattern of staining of these two antibodies and in particular the presence of breaks in type IV collagen and laminin at the dermo-epidermal junction are not specific for either benign or malignant melanocytic lesions and cannot be used as a diagnostic marker of invasive malignancy.

The differentiation between benign melanocytic naevi with a junctional component and true early invasive malignant melanoma is an important task for any pathologist. This can sometimes be difficult, particularly when a melanoma seems to have arisen in association with a benign pre-existing naevus, because the differentiation between benign naevus cells and infiltrating melanoma cells can be extremely subtle. The use of reticulin stains has been suggested in the past, and in future monoclonal antibodies which differentiate between benign and malignant cells of the melanocyte series may be of value, but these are not yet commercially available. We therefore carried out a study to look at two structural proteins normally present in the basement membrane, type IV collagen and laminin, to establish whether there are specific changes in the distribution of these proteins around melanocytic cells in benign and malignant melanocytic lesions.

\section{Material and methods}

In all, 154 lesions were studied. These were 24 junctional naevi, 21 compound naevi, 54 intradermal

Accepted for publication 13 July 1989 naevi, nine dysplastic naevi, and 46 malignant melanomas - 40 primary lesions and six cutaneous secondary deposits.

Dysplastic naevi were defined as compound naevi showing both architectural atypia in the form of lentiginous melanocytic dysplasia, and bridging between melanocyte nests at the dermo-epidermal junction, and cytological atypia.

Five $\mu \mathrm{m}$ thick sections were cut from stored blocks which had previously been fixed in $10 \%$ neutral buffered formalin and routinely processed in paraffin wax. Routine dewaxing and rehydration was carried out, and the sections were then exposed to $0 \cdot 1 \%$ trypsin (Difco) in $\mathbf{0 . 1 \%}$ calcium chloride solution at pH 7.2 for 30 minutes at $37^{\circ} \mathrm{C}$. Sections were then washed in water for two minutes, and normal swine serum diluted 1 in 5 in 0.05 molar TRIS buffered saline was then placed on the sections for 15 minutes at room temperature. This step reduced non-specific background staining. Sections were then incubated for one hour with primary antibody. The antibodies used were against type IV collagen (Sodichimie Basel) and against laminin (ICN Biomedical, Gibco, Paisley) both diluted 1 in 80 . After washing in TRIS buffered saline, sections were incubated for 30 minutes at room temperature with a 1 in 50 solution of swine anti-rabbit 


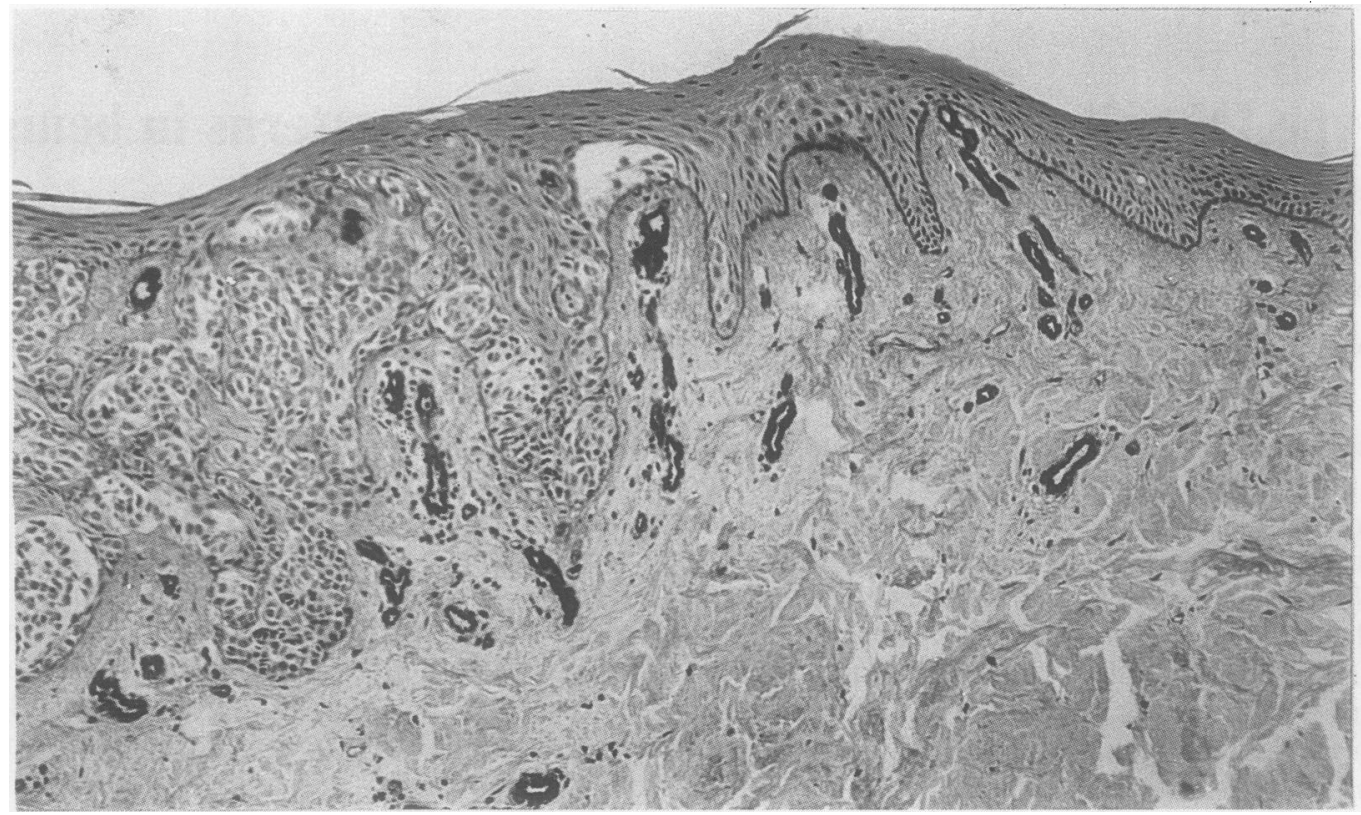

Fig 1 Edge of superficial spreading melanoma showing a continuous band of type IV collagen at right hand edge of picture under normal epidermis, but loss of this band at the left hand end under intraepidermal component of melanoma. Note in-built positive control provided by the blood vessel staining.

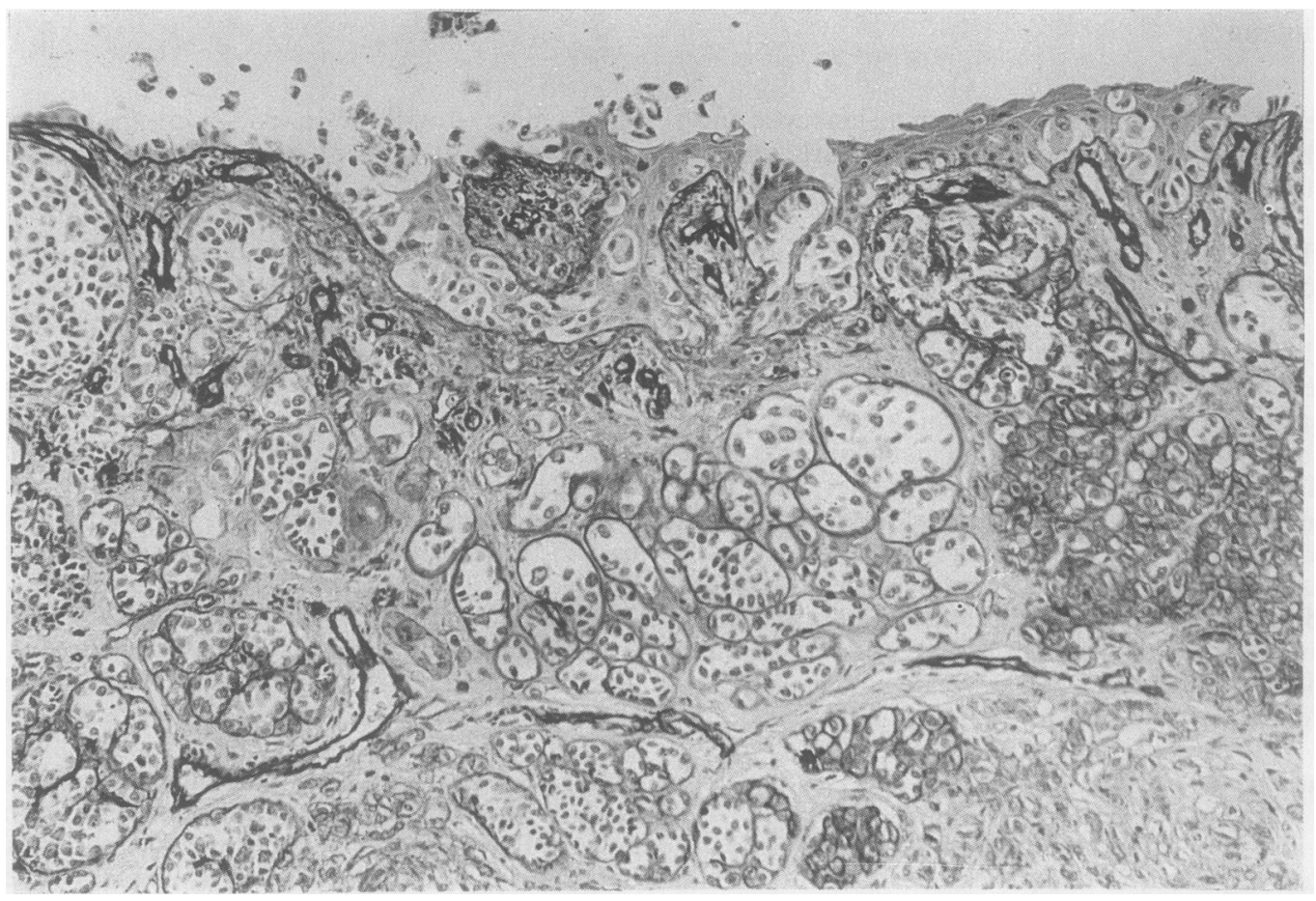

Fig 2 Invasive melanoma with a Breslow thickness of $1.3 \mathrm{~mm}$. Note total irregularity of the type IV collagen staining. There is absence of this staining at the dermo-epidermal junction but it is present around intradermal tumour cells, both singly and in clusters. 


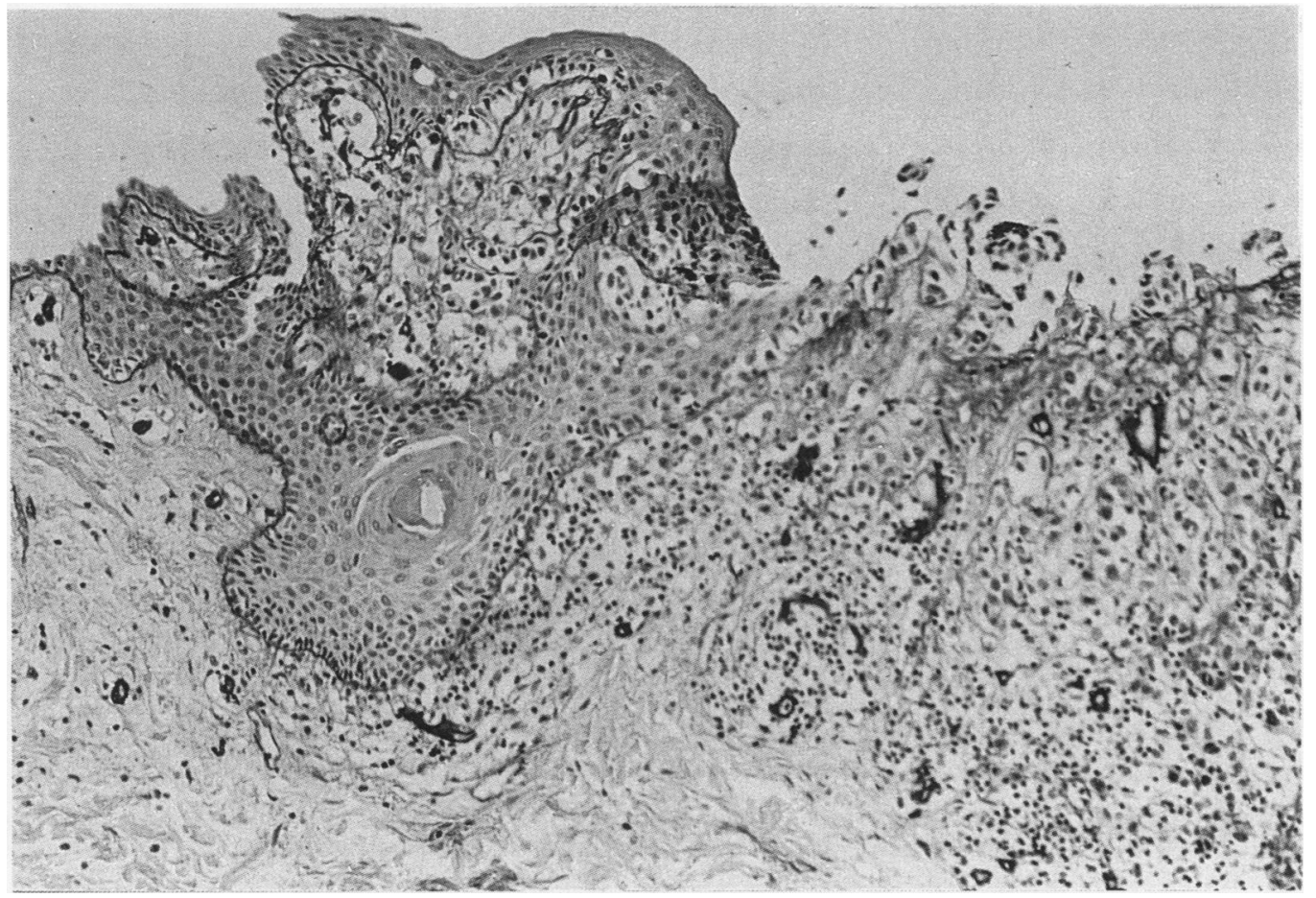

Fig 3 Note absence of type IV collagen staining adjacent to lymphocytic infiltrate in an early superficial spreading melanoma. A similar pattern is seen adjacent to similar infiltrates in dysplastic naevi.

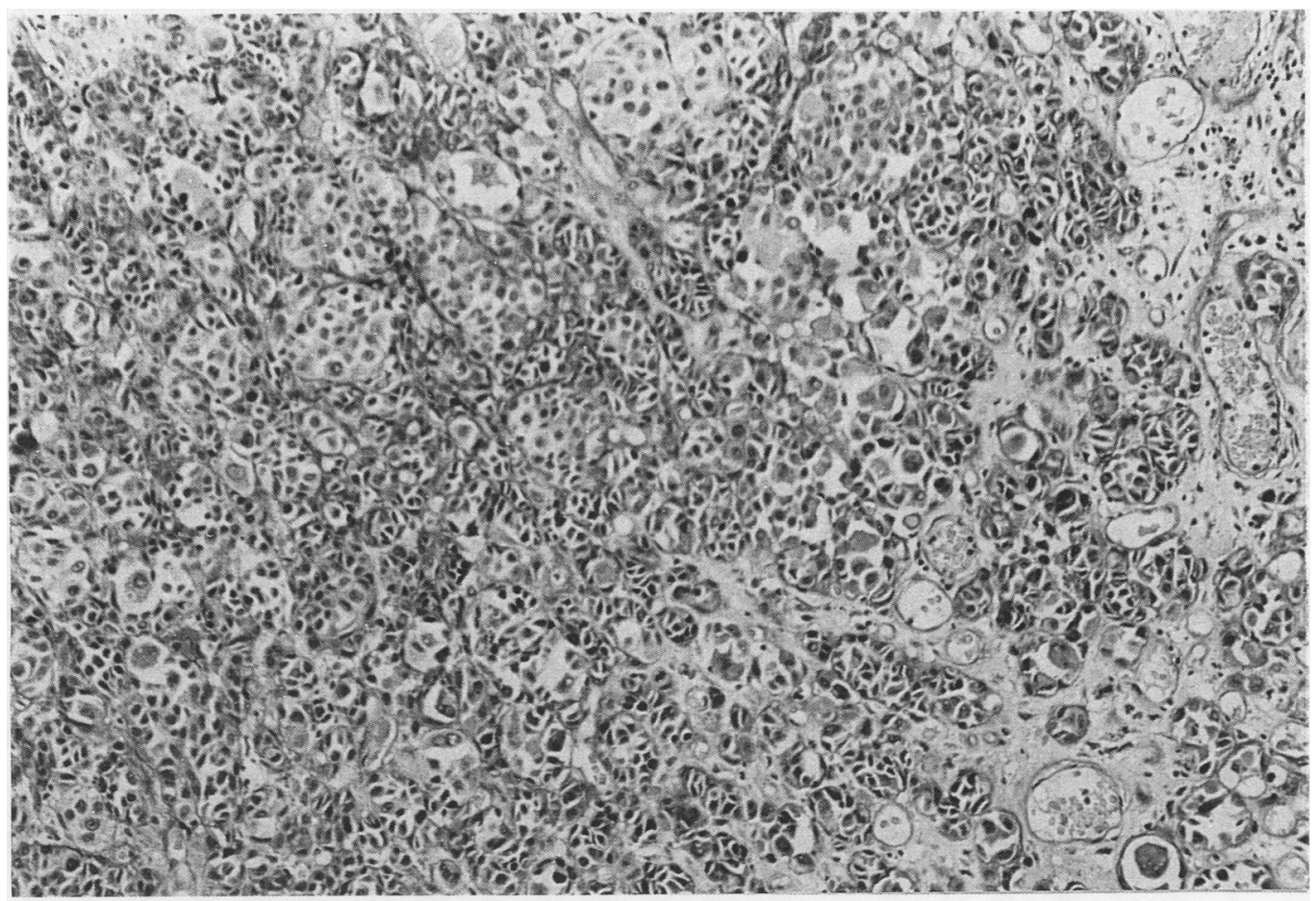

Fig 4 Secondary melanoma. Note membrane staining of type IV collagen around single cells and also some staining around clusters. There is also light cytoplasmic staining, suggesting type IV synthesis in the tumour cells. 


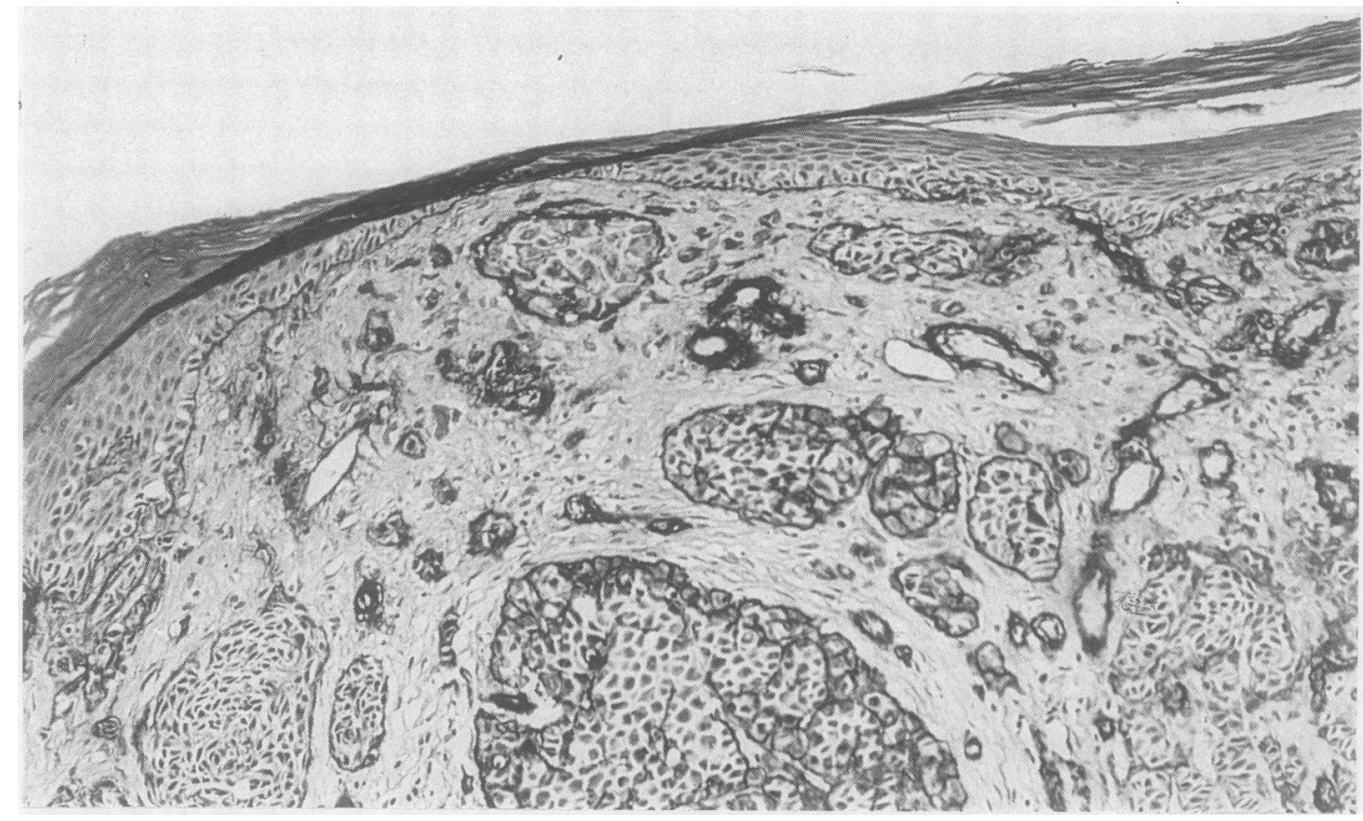

Fig 5 Benign compound naevus. Note variable and, in places, thin band of type IV collagen at the dermo-epidermal junction and heavy staining around intradermal naevus cells, both around individual cells and around nests.

antibody (Dako), and, after further washing, with 1 in 100 rabbit peroxidase-antiperoxidase complex in TRIS buffered saline. After further washing, the reaction product was developed by immersing the slides in $0.03 \%$ diaminobenzidine hydrochloride in TRIS buffer, $\mathrm{pH} \mathbf{7 \cdot 4}$, for five minutes at room temperature. Slides were then washed, dehydrated, and mounted.

\section{Results}

\section{MALIGNANT MELANOMAS}

In all the $\mathbf{4 0}$ examples of primary melanomas studied there was focal loss or pronounced thinning of the type IV collagen and laminin band found in the normal basement membrane. This was associated with areas of melanocytic proliferation at the dermo-epidermal junction (figs 1 and 2). As with the other types of lesions studied, the patterns of loss for type IV collagen and laminin were virtually identical, but in general a stronger reaction was seen with type IV collagen antibody. The loss of both proteins was particularly striking adjacent to areas of inflammatory infiltrate (fig 3).

In the dermis malignant melanoma cells frequently showed a surrounding mantle of type IV collagen and laminin, and this tended to be more pronounced around the more deeply invasive cells. In general a membrane staining pattern was seen but faint peripheral cytoplasmic staining was also seen. Thi staining around invasive tumour cells in the dermis was seen both around individual cells and arounct clusters of tumour cells. Because of this striking and unexpected pattern of staining around primary melanoma cells in the dermis, we examined six examples of secondary melanoma which had metastasised to the dermis. In all six cases this particular pattern of type IV collagen and laminin staining was seen in a distribution identical with that observed in the primary lesions (fig 4).

\section{BENIGN MELANOCYTIC NAEVI}

In the junctional naevi studied the pattern of loss was that of focal thinning of both type IV collagen and laminin in the basement membrane zone beneath areas of junctional activity. In some areas the band was completely lost, but staining was clearly seen on either side of the area of melanocytic proliferation. With compound naevi, a similar pattern was seen adjacent to the areas of junctional activity, with relatively little staining around cells in the papillary dermis (fig 5). Staining was, however, seen with both antibodies in the reticular dermis, and here the pattern was indistinguishable from that seen in the invasive component of the primary melanomas.

The intradermal naevi showed no abnormality of type IV collagen and laminin bands at the dermoepidermal junction but did show the same pattern of 
pericellular or membrane staining seen around dermal naevus cells seen in the intradermal component of the compound naevi.

The dysplastic naevi were all compound in character and showed features of type IV collagen and laminin staining which were identical with those of banal compound naevi, with the sole exception of more pronounced loss of staining seen adjacent to areas of lymphocytic infiltrate.

\section{Discussion}

This study has shown that there are consistent losses of type IV collagen and laminin from the basement membrane zone in areas adjacent to junctional melanocytic proliferation, but that these losses do not differentiate between benign junctional proliferation and early invasive primary melanoma. The pattern of staining around the deeper invasive cells and intradermal naevus cells is similar and suggests that the melanocytic cells in this site may actually synthesise type IV collagen and laminin. In vitro studies by Yaar Woodley and Gilchrest have previously shown that in culture this synthesis does take place.' Further evidence that such synthesis does occur comes from the observations of McArdle et al, who reported the presence of type IV collagen around a variety of tumour cells metastasising to the brain, including melanoma cells. ${ }^{2}$

Kirkham advocated the use of antibody to type VII collagen to identify melanomas thicker than $0.76 \mathrm{~mm}$ as in his experience breaks in the normal band of type VII collagen are seen only in melanomas thicker than this. ${ }^{3}$ In view of the fact that we observed both thinning and, at times, breaks in the basement membrane in benign naevi, it is clear that this observation does not apply to either or type IV collagen or laminin.

In an immunofluorescence study using similar antibodies Natali et al suggested that the presence of type IV collagen around clusters of intradermal cells rather than around single cells was commoner in melanoma than in naevi. ${ }^{4}$ We frequently observed staining around both single cells and clusters on the same section, from both benign and malignant lesions, and would not therefore regard this as a specific finding. This observation was also made by Steinback and Wasenius in a smaller series of pigmented lesions, using privately raised antibodies which are not commercially available. ${ }^{5}$

In conclusion, we did not find that the patterns of type IV collagen and laminin distribution differentiated between benign melanocytic naevi and early malignant melanoma and were struck by the apparent thinning and either non-synthesis or destruction of these proteins in the basement membrane area adjacent to unequivocally benign melanocytic naevi.

We acknowledge support from the Biomedical Research Committee, St Andrews House, Grant No K/MRS/50/C681.

\section{References}

1 Yaar M, Woodley DT, Gilchrest BA. Human naevocellular naevus cells are surrounded by basement membrane components. Lab Invest 1988;58:157-63.

2 McArdle JP, Muller K, Roff B, Murphy WH. Basal lamina redevelopment in tumours metastatic to brain. An immunoperoxidase study using an antibody to type IV collagen. Int J Cancer 1984;34:633-8.

3 Kirkham N, Gibson B, Leigh I, Coburn P, Darley CR, Price ML. Malignant melanoma: the differential diagnosis of thin lesions. $J$ Pathol 1987;152:220a.

4 Natali PG, Nicotra MR, Belocci M, Cavaliere R, Bigotti A. Distribution of laminin and collagen type IV in benign and malignant lesions of melanocytic origin. Int $J$ Cancer 1985;35:461-7.

5 Steniback F, Wasenius VM. Occurrence of basement membranes in pigment cell tumours of the skin: relation to cell type and clinical behaviour. J Cutan Pathol 1986;13:175-86.

Requests for reprints to: Professor Rona M MacKie, Department of Dermatology, University of Glasgow, Glasgow G12 8QQ Scotland. 